\title{
Predictive markers of capecitabine sensitivity identified from the expression profile of pyrimidine nucleoside-metabolizing enzymes
}

\author{
HIDEYUKI YASUNO $^{1}$, MITSUE KURASAWA ${ }^{1}$, MIEKO YANAGISAWA ${ }^{1}$, \\ YASUKO SATO $^{2}$, NAOKI HARADA ${ }^{1}$ and KAZUSHIGE MORI ${ }^{1}$ \\ ${ }^{1}$ Product Research Department and ${ }^{2}$ Discovery Pharmacology Department II, Kamakura Research Laboratories, \\ Chugai Pharmaceutical Co., Ltd., Kamakura 247-8530, Japan
}

Received June 26, 2012; Accepted September 14, 2012

DOI: $10.3892 /$ or.2012.2149

\begin{abstract}
Molecular markers predicting sensitivity to anticancer drugs are important and useful not only for selecting potential responders but also for developing new combinations. In the present study, we analyzed the difference in the sensitivity of xenograft models to capecitabine (Xeloda ${ }^{\circledR}$ ), 5'-deoxy-5-fluorouridine (5'-DFUR, doxifluridine, Furtulon ${ }^{\circledR}$ ) and 5-FU by comparing the mRNA levels of 12 pyrimidine nucleoside-metabolizing enzymes. Amounts of mRNA in the tumor tissues of 80 xenograft models were determined by real-time RT-PCR and mutual correlations were examined. A clustering analysis revealed that the 12 enzymes were divided into two groups; one group consisted of 8 enzymes, including orotate phosphoribosyl transferase (OPRT), TMP kinase (TMPK) and UMP kinase (UMPK), and was related to the de novo synthesis pathway for nucleotides, with mRNA expression levels showing significant mutual correlation. In the other group, 4 enzymes, including thymidine phosphorylase (TP) and dihydropyrimidine dehydrogenase (DPD), were involved in the salvage/degradation pathway of the nucleotides, and the mRNA levels of this group were dispersed more widely than that of the de novo group. Antitumor activity was assessed in 24 xenograft models for each drug. The antitumor activity of capecitabine and 5'-DFUR correlated significantly with the mRNA levels of TP and with the TP/DPD ratio, whereas the activity of 5-FU correlated significantly with OPRT, TMPK, UMPK and CD. In a stepwise regression analysis, TP and DPD were found to be independent predictive factors of sensitivity to capecitabine and 5'-DFUR, and UMPK was predictive of sensitivity to 5 -FU. These results indicate that the predictive factors for sensitivity to capecitabine and 5'-DFUR in xenograft
\end{abstract}

Correspondence to: Dr Kazushige Mori, Product Research Department, Kamakura Research Laboratories, Chugai Pharmaceutical Co., Ltd., 200 Kajiwara, Kamakura, Kanagawa 247-8530, Japan

E-mail: morikzs@chugai-pharm.co.jp

Key words: capecitabine, thymidine phosphorylase, dihydropyrimidine dehydrogenase, cytidine deaminase, thymidylate synthase, uridine phosphorylase models may be different from those for 5-FU, suggesting that these drugs may have different responders in clinical usage.

\section{Introduction}

The oral fluoropyrimidine capecitabine (Xeloda ${ }^{\circledR}$ ) is sequentially activated by three enzymes (Fig. 1), carboxylesterase, cytidine deaminase (CD) and thymidine phosphorylase (TP), and generates 5-FU selectively within tumor tissues (1-4). 5'-Deoxy-5-fluorouridine (5'-DFUR, doxifluridine, Furtulon ${ }^{\circledR}$ ) is an anticancer drug mainly used for patients with breast, colorectal and gastric cancers in Japan and the rest of East Asia, and is an intermediate of the capecitabine activation pathway, which is generated from 5'-DFUR by CD in the liver and in tumors (Fig. 1). 5-FU that has been delivered to the tumor tissues is inactivated by an enzyme, dihydropyrimidine dehydrogenase (DPD), which is expressed in the tumor tissues. We previously demonstrated that the sensitivity of human tumor xenografts to capecitabine and 5'-DFUR correlated significantly with the expression ratio of TP to DPD in tumor tissues (5). On the other hand, thymidylate synthase (TS) and other pyrimidine nucleoside-metabolizing enzymes are also reported as predictive factors for 5-FU sensitivity (6-8). In the present study, therefore, we investigated the relationship between the expression levels of 12 enzymes associated with pyrimidine nucleoside metabolisms (including TP, DPD, and TS) in tumor tissues and the sensitivity of the tumors to capecitabine and 5'-DFUR as compared with that to 5-FU in xenograft models.

Analyzing the factors that predict sensitivity to anticancer drugs is important and useful not only for selecting potential responders but also for developing new combinations. By selecting patients who will potentially respond to the therapy, it is possible to avoid the unnecessary treatment of patients who would not respond, thus sparing them the possible adverse effects. Furthermore, predictive factors render scientific rationales for new combination therapies, by which the sensitivity factor could be upregulated or the resistance factor be downregulated. In the present study, it was revealed that, of the pyrimidine nucleoside-metabolizing enzymes, only TP and DPD predicted a response to capecitabine and 5'-DFUR. Therefore, quantifying the expression levels of TP and DPD in tumor tissues makes it possible to select responders to 


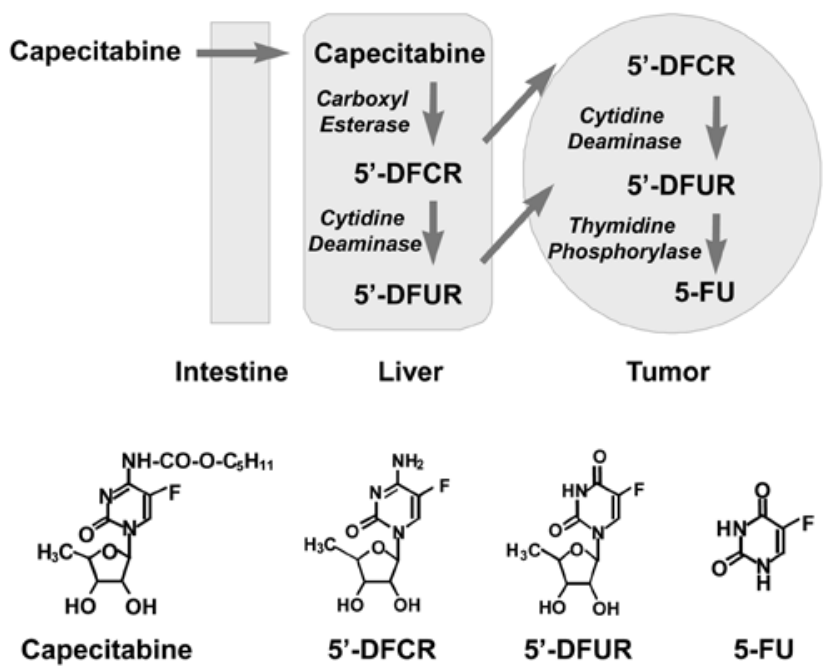

Figure 1. Activation pathway of capecitabine, and chemical structures of its metabolites. Capecitabine is activated to 5'-deoxy-5-fluorocytidine (5'-DFCR) by carboxyl esterase in the liver and to 5'-deoxy-5-fluorouridine (5'-DFUR) by cytidine deaminase in the liver and the tumor, and is finally converted to 5 -fluorouracil (5-FU) by thymidine phosphorylase in the tumor.

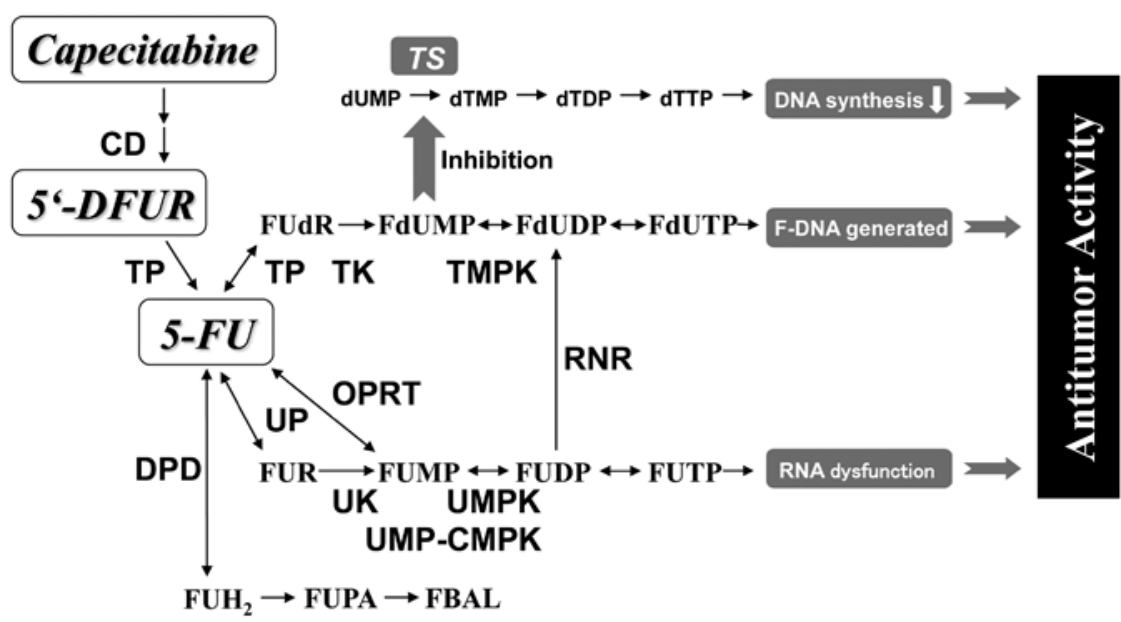

Figure 2. Metabolizing enzymes of capecitabine, 5'-DFUR and 5-FU. CD, cytidine deaminase; TP, thymidine phosphorylase; DPD, dihydropyrimidine dehydrogenase; UP, uridine phosphorylase; UK, uridine kinase; OPRT, orotate phosphoribosyl transferase; UMPK, UMP kinase; RNR, ribonucleotide reductase; TK, thymidine kinase; TMPK, TMP kinase; TS, thymidylate synthase.

capecitabine and 5'-DFUR. Moreover, investigating how combination partners regulate their expression during therapy would optimize combination therapies. Furthermore, capecitabine and 5'-DFUR may also be effective in 5-FU failure patients, even though these agents are 5-FU derivatives.

\section{Materials and methods}

Xenograft models. We used 80 xenograft models as listed in Table I. We determined the expression levels of mRNA in the tumor tissues from 80 xenograft models.

Quantification of mRNA levels in the tumor tissues from xenograft models. Fig. 2 illustrates how the pathway of capecitabine, 5'-DFUR, and 5-FU exerts its antitumor activity through pyrimidine nucleoside-metabolizing enzymes. We determined the mRNA expression levels of the pyrimidine nucleoside-metabolizing enzymes listed in Table II by a real- time RT-PCR method. Each mRNA level is expressed as the copy ratio to $10^{3}$ copies of GAPDH. Primers and hybridizationprobes specific to each mRNA are listed in Table III. RNR consists of a heterodimer of M1 and M2 subunits.

Total RNA was extracted from tumor tissues, which were stored in liquid nitrogen immediately after resection from nude mice, using Sepazol reagent (Nacalai Tesque, Inc., Kyoto, Japan) according to the manufacturer's recommendations. RNA quality was verified by electrophoresis through agarose gel and visualization with SYBR Green II (Molecular Probes, OR, USA). After treatment of RNA with DNase I (Invitrogen, Carlsbad, CA, USA), cDNA was synthesized from total RNA by using Superscript II RNase H-free reverse transcriptase (Invitrogen) and T7-poly dT (Qiagen, Tokyo, Japan).

The copy number of mRNA was determined using cDNA as a template in LightCycler ${ }^{\mathrm{TM}}$ (http://www.roche-appliedscience.com/lightcycler-online/). PCR was performed in LightCycler using specific primers (Table III). Specific signals 
Table I. List of xenograft models investigated.

\begin{tabular}{ll} 
Tumor type (no.) & \multicolumn{1}{c}{ Cell lines } \\
\hline Bladder (4) & 5637, RT-4, Scaber, T-24 \\
Breast (8) & BT474, KPL-4, MAXF401, MAXF449, MCF-7, MDA-MB-231, MDA-MB-468, MX-1 \\
Cervical (6) & C-33A, CAC-1, HT-3, ME-180, SIHA, Yumoto \\
Colorectal (16) & COLO201, COLO205, COLO320DM, CX-1, CXF280, DLD-1, HCT116, HCT15, HT-29, \\
Uterine (1) & LoVo, LS174T, LS411N, LS513, SW480, SW620, WiDr \\
Gastric (6) & GEC-59 \\
Hepatoma (4) & HLF, Huh-1, Huh-7, PLC/PRF/5 \\
Lung (12) & A549, Calu-1, Calu-3, Calu-6, LX-1, LXFL529, NCI-H292, NCI-H441, NCI-H569, PC10, \\
PC13, QG56 & A431, SEKI \\
Melanoma (2) & Nakajima, PA-1, SK-OV-3 \\
Ovarian (3) & AsPC-1, BxPC-3, Capan-1, Hs766T, MIAPaCa-2, Panc-1, SU86.86 \\
Pancreatic (7) & G-401, SK-ES-1, SK-N-AS, SK-PN-DW \\
Pediatric (4) & DU145, LNCaP-FGC, PC-3 \\
Prostate (3) & ACHN, SN-12C \\
Renal (2) &
\end{tabular}

The human cancer lines used were obtained from the following institutions: MCF-7, MDA-MB-231, COLO201, COLO320DM, HCT116, LoVo, LS174T, SW480, BxPC-3, MIAPaCa-2, Panc-1, and ACHN from Dainippon Pharmaceutical (Osaka, Japan); MKN28, MKN45, PC-10, PC-13, QG-56, and SEKI from Immunological Laboratories (Fujioka, Japan); CXF280, MAXF401, MAXF449, GXF97, and LXFL529 from Professor H.H. Fiebig (Friberg University, Triberg, Germany); T-24 from Dr H. Akaza (Tsukuba University, Tsukuba, Japan); KPL-4 from Dr J. Kurebayashi (Kawasaki Medical School, Okayama, Japan); MX-1, CX-1, and LX-1 from Cancer Chemotherapy Center (Japanese Foundation for Cancer Research, Tokyo, Japan); CAC-1 and HEC-59 from Dr H. Sasaki (The Jikei University School of Medicine, Tokyo, Japan); Yumoto from Dr H. Tokita (Chiba Cancer Center, Chiba, Japan); LS411N and LS513 from Dr L. Suardet (Swiss Institute for Experimental Cancer Research, Epalinges sur Lausanne); HLF, Huh-7, and PLC/PRF/5 from Dr E. Kataoka (National Institute of Hygienic Sciences, Tokyo, Japan); Huh-1 from the Institute of Cellular and Molecular Biology (Okayama University, Okayama, Japan); Nakajima from Dr S. Adachi (Niigata University, Niigata, Japan); SN-12C from Dr S. Naito (Kyushu University, Fukuoka, Japan); and the other cancer lines from the American Type Culture Collection (Rockville, MD). Scaber, MAXF401, MAXF449, MDA-MB-231, MX-1, Yumoto, CX-1, CXF280, GXF97, MKN28, LX-1, LXFL529, Nakajima, and SK-OV-3 were maintained by continuous passage in BALB/c-nu/nu mice. The other cancer lines were maintained in in vitro cultures.

from the PCR product were detected in LightCycler by two methods. One included the SYBR-Green method (LightCycler FastStart Master SYBR-Green, Roche Diagnostics, Mannheim, Germany) applied to UP, TK1, OPRT, UMP-CMPK, RNRM1, and RNRM2. The other method was the hybridization probe method (LightCycler FastStart DNA hybridization probes, Roche Diagnostics) applied to TP, DPD, CD, TS, UK, TMPK and UMPK. Specific hybridization probes are listed in Table III. GAPDH mRNA was also quantified by SYBR-Green method as an internal standard. Primer sequences for GAPDH were 5'-ACAGTCAGCCGCATCTTCTTTTG-3' (forward) and 5'-CCCACTTGATTTTGGAGGGATCT-3' (reverse). All primers and hybridization probes were synthesized by Qiagen and Nippon Gene Research (Sendai, Japan), respectively.

For a standard curve to quantify the copy number of the PCR signal template, we constructed plasmids containing each PCR product and determined the copy number in the plasmid preparation by $\mathrm{w} / \mathrm{v}$ concentration of plasmid. The mRNA expression levels of DPD were below the detection limit in 2 xenografts (DLD-1 and LS513). All other species of mRNA were able to be quantified in all xenografts.
Evaluation of the sensitivity of xenografts and treatment schedule. We determined the sensitivity of xenografts to capecitabine, 5'-DFUR and 5-FU in 24 models. To evaluate their sensitivity to the treatment, we calculated tumor growth inhibition (TGI) by using the following equation: TGI $\%=100 \mathrm{x}$ $(1-\Delta \mathrm{TVt} / \Delta \mathrm{TVc})$, where $\Delta \mathrm{TVt}$ is the tumor volume change after treatment with a drug and $\Delta \mathrm{TVc}$ is the tumor volume change after treatment with vehicle. The number of mice was 5-10 in the drug- and vehicle-treated groups for every xenograft model. Tumor volume (TV) was estimated by using the following equation: $\mathrm{TV}=a b^{2} / 2$, where $\mathrm{a}$ is the tumor length and $\mathrm{b}$ is the width.

The treatment schedule for each xenograft model is listed in Fig. 3A-C. A typical regimen is as follows: q7d or q5d for 3 weeks at the maximum tolerated dose (MTD). Treatment period and MTD were slightly different in some models because of the different susceptibility to toxicity of the treatment. The sensitivity to capecitabine, 5'-DFUR, and 5-FU is summarized in Fig. 3A, B and C, respectively.

Statistics. The mRNA levels in the tumor tissue were converted to logarithms before calculating the statistics. Correlation coef- 
Table II. List of pyrimidine nucleotide-metabolizing enzymes investigated.

\begin{tabular}{llll}
\hline mRNA & \multicolumn{1}{c}{ Enzyme name } & \multicolumn{1}{c}{ Synonyms } & EC \\
\hline TP & Thymidine phosphorylase & ECGF-1, PD-ECGF & 2.4 .2 .4 \\
DPD & Dihydropyrimidine dehydrogenase & & 1.3 .1 .2 \\
CD & Cytidine deaminase & & 3.5 .4 .5 \\
UP & Uridine phosphorylase & & 2.4 .2 .3 \\
TS & Thymidylate synthase & & 2.1 .1 .45 \\
TK1 & Thymidine kinase 1 & UCK2 & 2.7 .1 .21 \\
UK & Uridine kinase & UMP synthetase & 2.7 .1 .48 \\
OPRT & Orotate phosphorybosyl transferase & Thymidylate kinase & $4.1 .1 .23(2.4 .2 .10)$ \\
TMPK & TMP kinase & UCK1 & 2.7 .4 .9 \\
UMPK & UMP kinase & UMP-CMP kinase & 2.7 .4 .4 \\
UMP-CMPK & UMP kinase & & 2.7 .4 .14 \\
RNR M1 & Ribonucleotide reductase & & 1.17 .4 .1 \\
RNR M2 & Ribonucleotide reductase & & 1.17 .4 .1 \\
\hline
\end{tabular}

Table III. List of sequences of primers and hybridization-probes used.

\begin{tabular}{|c|c|c|c|}
\hline mRNA & $\begin{array}{l}\text { NCBI accession } \\
\text { no. }\end{array}$ & Primer Sequence & Hybridization-probe sequence \\
\hline $\mathrm{TP}$ & NM_001953 & Same sequences as primers in a commercial kit ${ }^{\mathrm{a}}$ & Same sequences as probes in a commercial kit ${ }^{\mathrm{a}}$ \\
\hline DPD & NM_000110 & Same sequences as primers in a commercial kit ${ }^{\mathrm{a}}$ & Same sequences as probes in a commercial kit ${ }^{\mathrm{a}}$ \\
\hline $\mathrm{CD}$ & NM_001785 & $\begin{array}{l}\text { 5'-CAGGAGGGGAGAATCTTCAAA } \\
\text { 5'-AGGCCCCACATGGAGAGATA }\end{array}$ & $\begin{array}{l}\text { 5'-ATCTGTGCTGAACGGACCGC-FITC } \\
\text { 5'-p-ATCCAGAAGGCCGTCTGAGAAGG-LCRed640 }\end{array}$ \\
\hline UP & NM_003364 & $\begin{array}{l}\text { 5'-TTTGTCGCCGGTGTCG } \\
\text { 5'-CGCGAGTCACCTCACCAA }\end{array}$ & SYBR Green method \\
\hline TS & NM_001071 & $\begin{array}{l}\text { 5'-GATCCCGAGACTTTTTGGACAGCC } \\
\text { 5'-CCATGTCTCCCGATCTCTGGTACA }\end{array}$ & $\begin{array}{l}\text { 5'-GAATCATCATGTGCGCTTGGAATC-FITC } \\
\text { 5'-p-AAGAGATCTTCCTCTGATGGCGCT-LCRed640 }\end{array}$ \\
\hline TK1 & NM_003258 & $\begin{array}{l}\text { 5'-TGCACACATGACCGGAACAC } \\
\text { 5'-GGCCTCGCAGAACTCCATG }\end{array}$ & SYBR Green method \\
\hline UK & NM_031432 & $\begin{array}{l}\text { 5'-GAGTTCTGCCTGCCGACAA } \\
\text { 5'-TGGTCCCCTGGCTCAGAA }\end{array}$ & $\begin{array}{l}\text { 5'-TGATCATCCCTAGAGGTGCAGATAATC-FITC } \\
\text { 5'-p-GGTGGCCATCAACCTCATCG-LCRed640 }\end{array}$ \\
\hline OPRT & NM_000373 & $\begin{array}{l}\text { 5'-GGGGGTGCCTCCTTATTG } \\
\text { 5'-TTGTGGGCTATTGTACTGTTGG }\end{array}$ & SYBR Green method \\
\hline TMPK & NM_012145 & $\begin{array}{l}\text { 5'-GCTGGGAACAAGTGCCGTT } \\
\text { 5'-GCCCACGTCTGGCTGTTTAC }\end{array}$ & $\begin{array}{l}\text { 5'-CGGTGAAGGCCACACCAGAA-FITC } \\
\text { 5'-p-ATGCGTATCTGTCCACGACGAGG-LCRed640 }\end{array}$ \\
\hline UMPK & NM_012474 & $\begin{array}{l}\text { 5'-GGAATTCTGCTTGCCAACAA } \\
\text { 5'-GCTGGGGTCCGATGGAG }\end{array}$ & $\begin{array}{l}\text { 5'-TGCAAATGGCACCGAGGAGG-FITC } \\
\text { 5'-p-TCCAATGGGCGGAGCTACAA-LCRed640 }\end{array}$ \\
\hline UMP-CMPK & NM_016308 & $\begin{array}{l}\text { 5'-GATTCTCCTCTGCTCTCCACG } \\
\text { 5'-TTGTCTGATCCATTTCCCTCTTT }\end{array}$ & SYBR Green method \\
\hline RNR M1 & NM_001033 & $\begin{array}{l}\text { 5'-TGGATGAGGTTTGGGGAGAG } \\
\text { 5'-GCATTTGATGGTTCCCAGGT }\end{array}$ & SYBR Green method \\
\hline RNR M2 & NM_001034 & $\begin{array}{l}\text { 5'-CACGGAGCCGAAAACTAAAG } \\
\text { 5'-ATTTGGAAGCCATAGAAACAGC }\end{array}$ & SYBR Green method \\
\hline
\end{tabular}

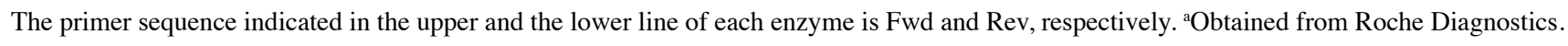

ficient (r) and significance of correlation (p) were calculated by a statistics software, StatView. Stepwise regression analysis, a type of multivariate analysis, was also performed by StatView.
Clustering analysis was performed by Eisen Gene Cluster using single linkage clustering method with similarity metric for uncentered correlation. 
A

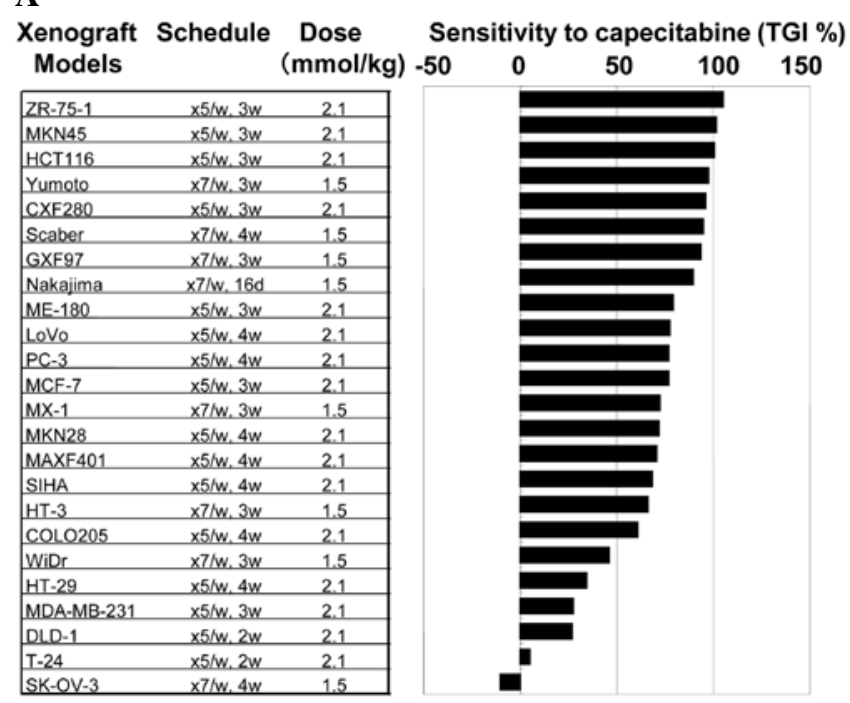

B

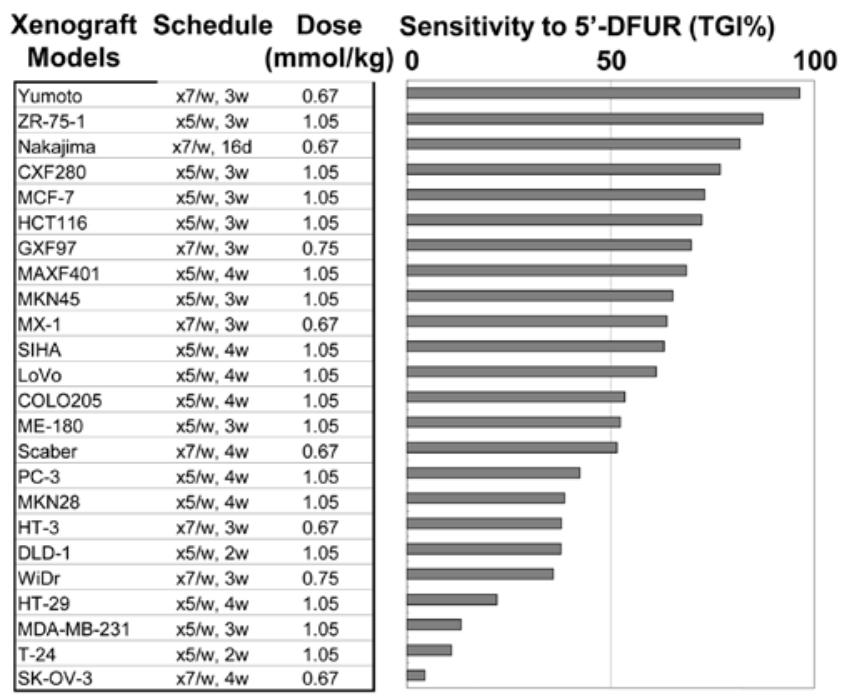

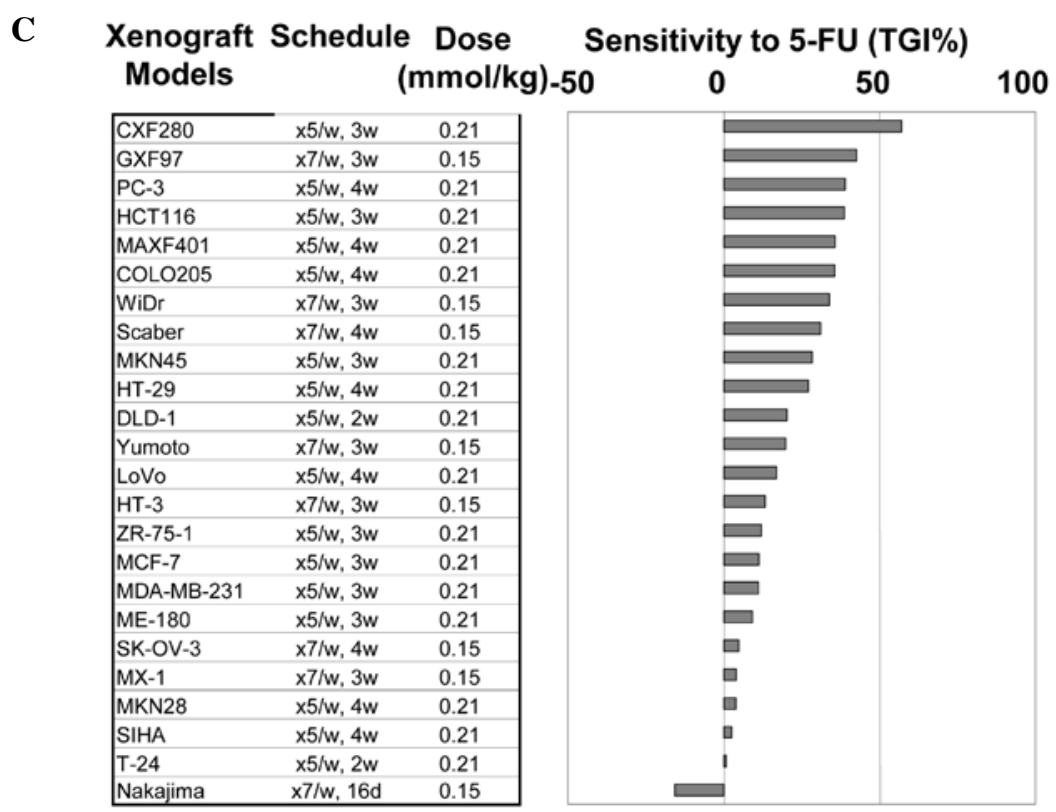

Figure 3. Summary of sensitivity to fluoropyrimidines with a list of treatment schedules and doses. Bar graph shows the tumor growth inhibition (TGI\%) of capecitabine (A), 5'-DFUR (B), and 5-FU (C) in 24 xenograft models. The left-hand lists summarize the treatment schedules and doses for capecitabine (A), 5'-DFUR (B), and 5-FU (C) in each xenograft model.

\section{Results}

mRNA expression levels of pyrimidine nucleoside-metabolizing enzymes. In the 80 models, the range of mRNA expression levels of TP, DPD, CD and UP was wider than that of TS, TK1, UK, OPRT, TMPK, UMPK, UMP-CMPK, RNRM1 and RNRM2 (Fig. 4). In the correlation analysis of these mRNA levels, the 12 types of enzymes were found to be divided into 2 groups. The first group included TS, TK1, UK, OPRT, TMPK, UMPK, UMP-CMPK, RNRM1 and RNRM2, and the expression levels of these mRNA were well correlated with each other. The second group included TP, DPD, UP, and $\mathrm{CD}$, and their mRNA levels were also somewhat correlated with each other (Fig. 5). However, there were only weak correlations between the expression levels of the first group and those of the second group (Fig. 5). The clustering analysis for the mRNA expression in tumors also revealed that the 12 types of pyrimidine nucleoside-metabolizing enzyme could be divided into 2 clusters (Fig. 6), one including TP, DPD, CD, and UP and the other including TS, TK1, UK, OPRT, TMPK, UMPK, UMP-CMPK, RNRM1 and RNRM2.

Correlation between $\mathrm{mRNA}$ levels and sensitivity to fluoropyrimidines in xenografts. Fig. 3 summarizes the sensitivity (TGI\%) of 24 xenografts to capecitabine, 5'-DFUR and 5-FU (Fig. 3A, B and C, respectively). Table IV shows the correlation between mRNA levels in tumor tissues and sensitivity to capecitabine, 5'-DFUR and 5-FU in xenograft models. In univariate analysis, it was demonstrated that the sensitivity to capecitabine correlated significantly with the mRNA level of TP positively, and with that of TS negatively. Similarly, the expression levels of TP were correlated positively, and those of DPD negatively, with the sensitivity to 5'-DFUR. The expression ratio of TP to DPD showed significant correlation with the 


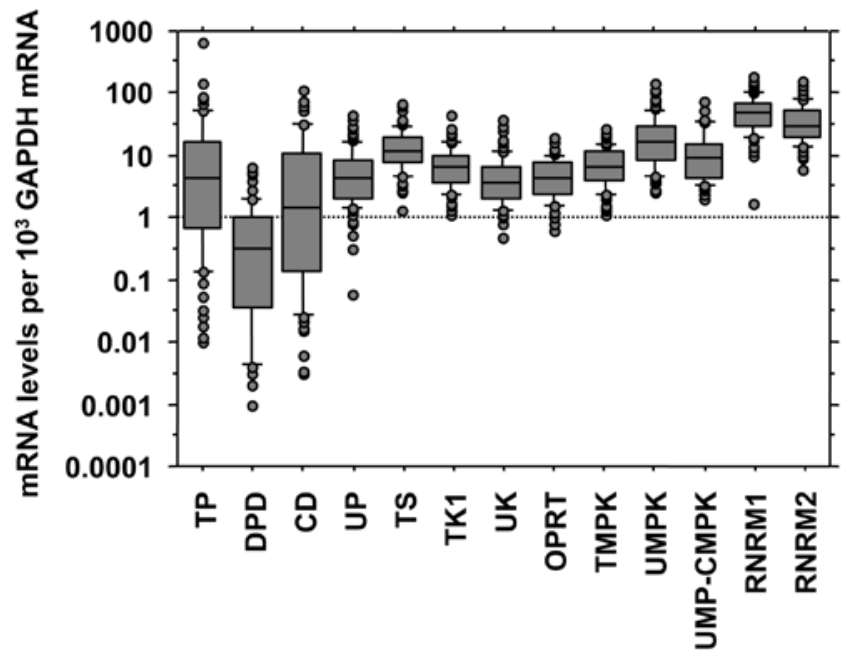

Figure 4. mRNA expression levels of pyrimidine nucleoside-metabolizing enzymes. The expression levels of mRNA in the tumor tissues were determined by real-time RT-PCR as described in Materials and methods. Boxes in the figure indicate the range where half of the data is included (between upper and lower quartile). A line in each box indicates the median value. Bars above and below each box indicate the range of lower $10 \%$ and upper $90 \%$, and closed circles indicate values beyond these ranges.

sensitivity to capecitabine and 5'-DFUR ( $\mathrm{r}=0.746$ and $\mathrm{r}=0.798$, respectively). On the other hand, the sensitivity to 5-FU showed a significant positive correlation with CD, OPRT, TMPK, UMPK and UMP-CMPK but not with mRNA levels of TP, DPD and TS, and the TP/DPD ratio did not show significant correlation with the sensitivity to 5-FU. As there was a significant correlation among several of the expression levels of mRNAs, we next analyzed the independent explanatory variable for the drug sensitivity by a stepwise regression method, a type of multivariate analysis. In the results, the mRNA levels of TP and DPD emerged as independent explanatory variables for the sensitivity to capecitabine. The same was equally true of 5'-DFUR. On the other hand, the independent explanatory variable for that of 5-FU was the expression level of UMPK.

\section{Discussion}

De novo versus salvage. In 80 xenograft models listed in Table I, we determined the mRNA levels in the tumor tissues of pyrimidine nucleoside-metabolizing enzymes listed in Table II. Fig. 4 summarizes the mRNA expression levels of each enzyme in the tumor tissues: 13 species of mRNA of 12 types of enzymes (RNR consists of heterodimer of M1 and M2 subunits). These data demonstrate that the deviation of mRNA expression levels of TP, DPD, CD, and UP was larger than that of the other pyrimidine nucleoside-metabolizing enzymes: TS, TK1, UK, OPRT, TMPK, UMPK, UMP-CMPK, RNRM1, and RNRM2. The former set of enzymes can be classified into the salvage/degradation pathway of pyrimidine nucleotide metabolism, and the latter set into the de novo synthesis pathway. The de novo pathway enzymes would be essential for cell proliferation, whereas the salvage enzymes may not necessarily be required. Therefore, it is probable that the expression of the de novo enzymes is tightly regulated within appropriate levels, whereas that of salvage enzymes is not. Indeed, the expression levels of the de novo enzymes, TS,
Table IV. Correlation between mRNA levels and sensitivity to fluoropyrimidines.

\begin{tabular}{|c|c|c|c|}
\hline \multirow[b]{2}{*}{ mRNA } & \multicolumn{3}{|c|}{ Correlation with sensitivity to } \\
\hline & Capecitabine & 5'-DFUR & $5-\mathrm{FU}$ \\
\hline \multirow[t]{2}{*}{$\mathrm{TP}$} & 0.587 & 0.463 & 0.187 \\
\hline & 0.0021 & 0.0216 & 0.3848 \\
\hline \multirow[t]{2}{*}{ DPD } & -0.513 & -0.613 & -0.221 \\
\hline & 0.0113 & 0.0014 & 0.3142 \\
\hline \multirow[t]{2}{*}{$\mathrm{CD}$} & 0.016 & -0.227 & 0.470 \\
\hline & 0.9403 & 0.2907 & 0.0196 \\
\hline \multirow[t]{2}{*}{ UP } & -0.080 & -0.162 & 0.290 \\
\hline & 0.7131 & 0.4527 & 0.1712 \\
\hline \multirow[t]{2}{*}{ TS } & -0.260 & -0.295 & 0.303 \\
\hline & 0.2228 & 0.1640 & 0.1513 \\
\hline \multirow[t]{2}{*}{ TK1 } & -0.014 & -0.115 & 0.381 \\
\hline & 0.9498 & 0.5957 & 0.0659 \\
\hline \multirow[t]{2}{*}{ UK } & -0.028 & -0.019 & 0.144 \\
\hline & 0.8968 & 0.9298 & 0.5067 \\
\hline \multirow[t]{2}{*}{ OPRT } & -0.077 & -0.170 & 0.434 \\
\hline & 0.7240 & 0.4327 & $\mathbf{0 . 0 3 3 8}$ \\
\hline \multirow[t]{2}{*}{ TMPK } & -0.273 & -0.281 & 0.431 \\
\hline & 0.1996 & 0.1850 & 0.0348 \\
\hline \multirow[t]{2}{*}{ UMPK } & -0.032 & 0.011 & 0.554 \\
\hline & 0.8830 & 0.9586 & 0.0042 \\
\hline \multirow[t]{2}{*}{ UMP-CMPK } & -0.138 & -0.234 & 0.458 \\
\hline & 0.5235 & 0.2756 & 0.0235 \\
\hline \multirow[t]{2}{*}{ RNRM1 } & -0.163 & -0.128 & 0.286 \\
\hline & 0.4496 & 0.5560 & 0.1775 \\
\hline \multirow[t]{2}{*}{ RNRM2 } & -0.052 & 0.008 & 0.343 \\
\hline & 0.8108 & 0.9720 & 0.1014 \\
\hline \multirow[t]{2}{*}{ TP/DPD } & 0.746 & 0.798 & 0.324 \\
\hline & $<0.0001$ & $<0.0001$ & 0.1328 \\
\hline
\end{tabular}

Correlation between mRNA levels in the tumor tissues and the sensitivity of the xenografts to capecitabine, 5'-DFUR, and 5-FU were respectively analyzed as described in Materials and methods. Correlation efficient (r) and significance (p) is indicated in the upper line and lower line for each enzyme, respectively. Correlation with $\mathrm{p}<0.05$ is in bold.

TK1, UK, OPRT, TMPK, UMPK, UMP-CMPK, RNRM1, and RNRM2, correlated with each other significantly, whereas those of the salvage enzymes, TP, DPD, CD, and UP, were found to correlate to a lesser extent. Furthermore, the salvage enzymes and the de novo enzymes were respectively categorized in 2 clusters by a clustering analysis of the mRNA expression (Fig. 6). These clusters suggest that the regulation of enzymes in the de novo pathway is independent of that in the salvage. 


\begin{tabular}{|c|c|c|c|c|c|c|c|c|c|c|c|c|}
\hline mRNA & DPD & $C D$ & UP & TS & TK1 & UK & OPRT & TMPK & UMPK & $\begin{array}{l}\text { UMP- } \\
\text { CMPK }\end{array}$ & RNRM1 & RNRM2 \\
\hline \multirow[t]{12}{*}{ TP } & $\begin{array}{c}0.309 \\
0.0056\end{array}$ & $\begin{array}{c}0.483 \\
<0.0001\end{array}$ & $\begin{array}{c}0.427 \\
<0.0001\end{array}$ & $\begin{array}{l}-0.227 \\
0.0431\end{array}$ & $\begin{array}{c}0.040 \\
0.7243\end{array}$ & $\begin{array}{c}0.078 \\
0.4948\end{array}$ & $\begin{array}{c}0.116 \\
0.3074\end{array}$ & $\begin{array}{c}0.117 \\
0.3004\end{array}$ & $\begin{array}{l}-0.016 \\
0.8890\end{array}$ & $\begin{array}{c}0.336 \\
0.0022\end{array}$ & $\begin{array}{c}0.042 \\
0.7102\end{array}$ & $\begin{array}{c}0.089 \\
0.4331\end{array}$ \\
\hline & DPD & $\begin{array}{c}0.227 \\
0.0454\end{array}$ & $\begin{array}{c}0.335 \\
0.0025\end{array}$ & $\begin{array}{c}0.206 \\
0.0698\end{array}$ & $\begin{array}{c}0.057 \\
0.6182\end{array}$ & $\begin{array}{c}0.187 \\
0.1021\end{array}$ & $\begin{array}{c}0.082 \\
0.4756\end{array}$ & $\begin{array}{c}0.301 \\
0.0071\end{array}$ & $\begin{array}{c}0.001 \\
0.9911\end{array}$ & $\begin{array}{c}0.266 \\
0.0180\end{array}$ & $\begin{array}{c}0.049 \\
0.6694\end{array}$ & $\begin{array}{c}0.190 \\
0.0961\end{array}$ \\
\hline & & $C D$ & $\begin{array}{c}0.537 \\
<0.0001\end{array}$ & $\begin{array}{c}0.006 \\
0.9592\end{array}$ & $\begin{array}{c}0.385 \\
0.0004\end{array}$ & $\begin{array}{l}-0.011 \\
0.9200\end{array}$ & $\begin{array}{c}0.276 \\
0.0130\end{array}$ & $\begin{array}{c}0.344 \\
0.0016\end{array}$ & $\begin{array}{c}0.083 \\
0.4660\end{array}$ & $\begin{array}{c}0.470 \\
<0.0001\end{array}$ & $\begin{array}{c}0.237 \\
0.0341\end{array}$ & $\begin{array}{c}0.131 \\
0.2459\end{array}$ \\
\hline & & & UP & $\begin{array}{c}0.177 \\
0.1165\end{array}$ & $\begin{array}{c}0.229 \\
0.0411\end{array}$ & $\begin{array}{c}0.159 \\
0.1589\end{array}$ & $\begin{array}{c}0.203 \\
0.0711\end{array}$ & $\begin{array}{c}0.294 \\
0.0080\end{array}$ & $\begin{array}{c}0.103 \\
0.3653\end{array}$ & $\begin{array}{c}0.395 \\
0.0002\end{array}$ & $\begin{array}{c}0.253 \\
0.0231\end{array}$ & $\begin{array}{c}0.183 \\
0.1047\end{array}$ \\
\hline & & & & TS & $\begin{array}{c}0.436 \\
<0.0001\end{array}$ & $\begin{array}{c}0.631 \\
<0.0001\end{array}$ & $\begin{array}{c}0.511 \\
<0.0001\end{array}$ & $\begin{array}{c}0.600 \\
<0.0001\end{array}$ & $\begin{array}{c}0.582 \\
<0.0001\end{array}$ & $\begin{array}{c}0.366 \\
0.0008\end{array}$ & $\begin{array}{c}0.538 \\
<0.0001\end{array}$ & $\begin{array}{c}0.674 \\
<0.0001\end{array}$ \\
\hline & & & & & TK1 & $\begin{array}{c}0.455 \\
<0.0001\end{array}$ & $\begin{array}{c}0.648 \\
<0.0001\end{array}$ & $\begin{array}{c}0.542 \\
<0.0001\end{array}$ & $\begin{array}{c}0.434 \\
<0.0001\end{array}$ & $\begin{array}{c}0.498 \\
<0.0001\end{array}$ & $\begin{array}{c}0.492 \\
<0.0001\end{array}$ & $\begin{array}{c}0.407 \\
0.0002\end{array}$ \\
\hline & & & & & & UK & $\begin{array}{c}0.691 \\
<0.0001\end{array}$ & $\begin{array}{c}0.658 \\
<0.0001\end{array}$ & $\begin{array}{c}0.631 \\
<0.0001\end{array}$ & $\begin{array}{c}0.468 \\
<0.0001\end{array}$ & $\begin{array}{c}0.503 \\
<0.0001\end{array}$ & $\begin{array}{c}0.619 \\
<0.0001\end{array}$ \\
\hline & & & & & & & OPRT & $\begin{array}{c}0.732 \\
<0.0001\end{array}$ & $\begin{array}{c}0.703 \\
<0.0001\end{array}$ & $\begin{array}{c}0.679 \\
<0.0001\end{array}$ & $\begin{array}{c}0.528 \\
<0.0001\end{array}$ & $\begin{array}{c}0.589 \\
<0.0001\end{array}$ \\
\hline & & & & & & & & TMPK & $\begin{array}{c}0.551 \\
<0.0001\end{array}$ & $\begin{array}{c}0.588 \\
<0.0001\end{array}$ & $\begin{array}{c}0.471 \\
<0.0001\end{array}$ & $\begin{array}{c}0.547 \\
<0.0001\end{array}$ \\
\hline & & & & & & & & & UMPK & $\begin{array}{c}0.605 \\
<0.0001\end{array}$ & $\begin{array}{l}0.360 \\
0.0010\end{array}$ & $\begin{array}{c}0.718 \\
<0.0001\end{array}$ \\
\hline & & & & & & & & & & -СMPK & $\begin{array}{c}0.266 \\
0.0169\end{array}$ & $\begin{array}{c}0.494 \\
<0.0001\end{array}$ \\
\hline & & & & & & & & & & & RNRM1 & $\begin{array}{c}0.468 \\
<0.0001\end{array}$ \\
\hline
\end{tabular}

Figure 5. Correlation among the expression levels of mRNAs. Correlation among the mRNA expression levels of pyrimidine nucleoside-metabolizing enzymes in the tumor tissues of 80 xenograft models was analyzed as described in Materials and methods. Correlation efficient $(r)$ and significance $(p)$ is indicated in the upper line and lower line of each box, respectively. Correlation with $\mathrm{p}<0.0001$ is in bold.

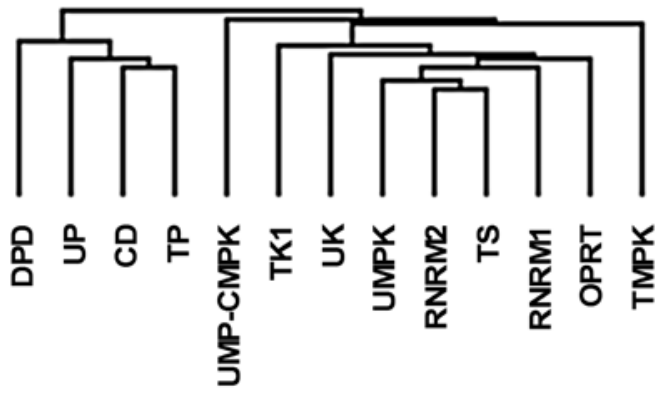

Figure 6. Clustering analysis for mRNA expression of each enzyme. The dendrogram of enzymes was drawn using the Eisen Gene Cluster software.

$T P$ and DPD as predictive factors for capecitabine and 5 '-DFUR. Tumor levels of TP and DPD have been found to correlate with the sensitivity of xenografts to capecitabine and 5'-DFUR (5). Since both drugs exert antitumor activity after being converted to 5-FU (Fig. 2), the other enzymes metabolizing 5-FU may influence the antitumor activities of these drugs. Therefore, we analyzed the relationship between the sensitivity of xenografts to the fluoropyrimidines (capecitabine, 5'-DFUR and 5-FU) and the tumor levels of mRNA of the pyrimidine nucleoside-metabolizing enzymes, including TP and DPD. The sensitivity of xenografts to capecitabine correlated significantly with TP, CD, and TS, and that to 5'-DFUR correlated significantly with TP and DPD. The expression ratio of TP to DPD correlated well with capecitabine and 5'-DFUR. On the other hand, the expression levels of OPRT, TMPK, UMPK, UMP-CMPK, and CD correlated significantly with the sensitivity of xenografts to 5-FU, but those of TP and DPD did not. As stated above, the expression levels of these enzymes significantly correlated with each other in some cases. Therefore, we analyzed the independent explanatory factors for the sensitivity of xenografts by using a stepwise regression method, a type of multivariate analysis. Consequently, it was revealed that the independent explanatory factors for the sensitivity to capecitabine and 5'-DFUR were the mRNA levels of TP and DPD, whereas that to 5-FU was only UMPK. In the present study, it was demonstrated that out of 12 types of pyrimidine nucleoside-metabolizing enzymes, TP and DPD had the most influence on the efficacy of capecitabine and 5'-DFUR. Therefore, it may be possible to predict the response to capecitabine and 5'-DFUR by quantifying only the expression levels of TP and DPD. On the other hand, the predictive factor for 5-FU was different from capecitabine and 5'-DFUR. Therefore, although they are categorized as 5-FU derivatives, capecitabine and 5'-DFUR may be effective even in 5-FU failure.

$T P$ upregulation. In the present study, the tumors with low expression levels of TP did not respond well to capecitabine and 5'-DFUR. However, these tumors would respond to these chemotherapeutics if TP expression could be upregulated in the tumor tissues. TP expression in the tumor tissues of animal models was reported to be upregulated after treatment with antitumor drugs $(9,10)$ or irradiation (11). In clinical studies, it has also been reported that the TP levels in breast carcinoma cells were augmented after docetaxel was administered as a neoadjuvant chemotherapy in advanced breast cancer patients (12). Toi and Ohni (13) reported that a marked increase in TP expression was found in tumors from patients with operable breast cancer who were treated with anthracycline and cyclophosphamide in combination (AC and EC) or with docetaxel. Therefore, the combination therapy of capecitabine/5'-DFUR with anticancer drugs that induce TP in the tumor tissue would be a promising option for cancer treatment. A phase III study 
demonstrated that combination therapy of capecitabine with docetaxel is more effective than single-agent docetaxel in patients with advanced breast cancer (14).

Clinical investigation of predictive factors TP and DPD for individualized therapy. In clinical studies, the tumor levels of TP and DPD have been reported to correlate with the efficacy of capecitabine and 5'-DFUR (15-17). TP and DPD may be predictive for the response to these chemotherapeutics in many types of cancer. Further study validating is needed to confirm the possibility that tumors with a high TP or TP/DPD ratio would respond well to capecitabine and 5'-DFUR. The expression levels of TP and DPD in tumor tissues can be determined by measuring mRNA levels as shown in the present study, and also by immunohistochemistry and ELISA (18-21). Currently, various methods are available to determine the expression levels of TP and DPD, as circumstances demand.

In conclusion, the present study demonstrated that of the major pyrimidine nucleoside-metabolizing enzymes, only TP and DPD were predictive factors in tumor tissues for the sensitivity to capecitabine and 5'-DFUR in xenograft models. This suggests the possibility of selecting patients who would potentially respond to capecitabine and 5'-DFUR by determining TP and DPD levels in tumor tissues. In addition, our study demonstrated that the predictive factor for capecitabine and 5'-DFUR was different from that for 5-FU in xenograft models. It means that capecitabine and 5'-DFUR would be effective even in 5-FU failure patients. The combination therapies of capecitabine with various drugs are now being clinically evaluated in patients for whom regimens including 5-FU have failed.

\section{References}

1. Miwa M, Ura M, Nishida M, Sawada N, Ishikawa T, Mori K Shimma N, Umeda I and Ishitsuka H: Design of a novel oral fluoropyrimidine carbamate, capecitabine, which generates 5 -fluorouracil selectively in tumours by enzymes concentrated in human liver and cancer tissue. Eur J Cancer 34: 1274-1281, 1998.

2. Ishikawa T, Utoh M, Sawada N, Nishida M, Fukase Y, Sekiguchi F and Ishitsuka $\mathrm{H}$ : Tumor selective delivery of 5-fluorouracil by capecitabine, a new oral fluoropyrimidine carbamate, in human cancer xenografts. Biochem Pharmacol 55: 1091-1097, 1998.

3. Schuller J, Cassidy J, Dumont E, Roos B, Durston S, Banken L, Utoh M, Mori K, Weidekamm E and Reigner B: Preferential activation of capecitabine in tumors following oral administration to colorectal cancer patients. Cancer Chemother Pharmacol 45: 291-297, 2000

4. Pentheroudakis G and Twelves C: The rational development of capecitabine from the laboratory to the clinic. Anticancer Res 22: 3589-3596, 2002.

5. Ishikawa T, Sekiguchi F, Fukase $\mathrm{Y}$, Sawada $\mathrm{N}$ and Ishitsuka H: Positive correlation between the efficacy of capecitabine and doxifluridine and the ratio of thymidine phosphorylase to dihydropyrimidine dehydrogenase activities in tumors in human cancer xenografts. Cancer Res 58: 685-690, 1998.

6. Aschele C, Sobrero A, Faderan MA and Bertino JR: Novel mechanism(s) of resistance to 5-fluorouracil in human colon cancer (HCT-8) sublines following exposure to two different clinically relevant dose schedules. Cancer Res 52: 1855-1864, 1992.
7. Aschele C, Lonardi S and Monfardini S: Thymidylate synthase expression as a predictor of clinical response to fluoropyrimidinebased chemotherapy in advanced colorectal cancer. Cancer Treat Rev 28: 27-47, 2002

8. Kubota T, Watanabe M, Otani Y, Kitajima M and Fukushiuma M: Different pathways of 5-fluorouracil metabolism after continuous venous or bolus injection in patients with colon carcinoma: possible predictive value of thymidylate synthetase mRNA and ribonucleotide reductase for 5-fluorouracil sensitivity. Anticancer Res 22: 3537-3540, 2002.

9. Sawada N, Ishikawa T, Fukase Y, Nishida M, Yoshikubo T and Ishitsuka H: Induction of thymidine phosphorylase activity and enhancement of capecitabine efficacy by taxol/taxotere in human cancer xenografts. Clin Cancer Res 4: 1013-1019, 1998.

10. Endo M, Shinbori N, Fukase Y, Sawada N, Ishikawa T, Ishitsuka H and Tanaka Y: Induction of thymidine phosphorylase expression and enhancement of efficacy of capecitabine or 5'-deoxy-5-fluorouridine by cyclophosphamide in mammary tumor models. Int $\mathbf{J}$ Cancer 83: 127-134, 1999.

11. Sawada N, Ishikawa T, Sekiguchi F, Tanaka Y and Ishitsuka H: $\mathrm{X}$-ray irradiation induces thymidine phosphorylase and enhances the efficacy of capecitabine (Xeloda) in human cancer xenografts. Clin Cancer Res 5: 2948-2953, 1999.

12. Kurosumi M, Tabei T, Suemasu K, Inoue K, Kusawake T, Sugamata N and Higashi Y: Enhancement of immunohistochemical reactivity for thymidine phosphorylase in breast carcinoma cells after administration of docetaxel as a neoadjuvant chemotherapy in advanced breast cancer patients. Oncol Rep 7: 945-948, 2000.

13. Toi M and Ohno S: The role of thymidine phosphorylase (TP) in choosing FU/capecitabine chemotherapy. Proc Am Soc Clin Oncol 22: 137, 2003.

14. O'Shaughnessy J, Miles D, Vukelja S, Moiseyenko V, Ayoub JP, Cervantes G, Fumoleau P, Jones S, Lui WY, Mauriac L, et al: Superior survival with capecitabine plus docetaxel combination therapy in anthracycline-pretreated patients with advanced breast cancer: phase III trial results. J Clin Oncol 20: 2812-2823, 2002.

15. Terashima M, Fujiwara H, Takagane A, Abe K, Araya M, Irinoda T, Yonezawa H, Nakaya T, Oyama K, Takahashi M, and Saito K: Role of thymidine phosphorylase and dihydropyrimidine dehydrogenase in tumour progression and sensitivity to doxifluridine in gastric cancer patients. Eur J Cancer 38: 2375-2381, 2002.

16. Nishimura G, Terada I, Kobayashi T, Ninomiya I, Kitagawa $H$, Fushida S, Fujimura T, Kayahara M, Shimizu K, Ohta T and Miwa K: Thymidine phosphorylase and dihydropyrimidine dehydrogenase levels in primary colorectal cancer show a relationship to clinical effects of 5'-deoxy-5-fluorouridine as adjuvant chemotherapy. Oncol Rep 9: 479-482, 2002.

17. Ishii R, Takiguchi N, Oda K, Koda K and Miyazaki M: Thymidine phosphorylase expression is useful in selecting adjuvant chemotherapy for stage III gastric cancer. Int J Oncol 19: 717-722, 2001

18. Nishida M, Hino A, Mori K, Matsumoto T, Yoshikubo T and Ishitsuka H: Preparation of anti-human thymidine phosphorylase monoclonal antibodies useful for detecting the enzyme levels in tumor tissues. Biol Pharm Bull 19: 1407-1411, 1996

19. Kono T, Nishida M, Inagaki N, Tanaka Y, Yoneda M and Kasai S: Development and characterization of 1C6-203, a new monoclonal antibody specific to human thymidine phosphorylase. J Histochem Cytochem 49: 131-138, 2001.

20. Komuro Y, Watanabe T, Tsuno N, Kitayama J, Inagaki N, Nishida M and Nagawa H:The usefulness of immunohistochemical evaluation of dihydropyrimidine dehydrogenase for rectal cancer treated with preoperative radiotherapy. Hepatogastroenterology 50: 906-911, 2003.

21. Mori K, Hasegawa M, Nishida M, Toma H, Fukuda M, Kubota T, Nagasue N, Yamana H, Hirakawa-YS Chung K, Ikeda T, et al: Expression levels of thymidine phosphorylase and dihydropyrimidine dehydrogenase in various human tumor tissues. Int $\mathbf{J}$ Oncol 17: 33-38, 2000. 